\title{
The Effect of a Multi-strain Probiotic on the Symptoms and Small Intestinal Bacterial Overgrowth in Constipation-predominant Irritable Bowel Syndrome: A Randomized, Simple-blind, Placebo-controlled Trial
}

\author{
Ivashkin V., Drapkina O., Poluektova Ye., Kuchumova S. , Sheptulin A., Shifrin O. \\ Department of Internal Disease Propaedeutics, Gastroenterology and Hepatology, I.M.Sechenov First Moscow State Medical \\ University, Moscow, Russia \\ *Corresponding author: svetlana.kuchumova@gmail.com
}

Received March 01, 2015; Revised April 03, 2015; Accepted April 07, 2015

\begin{abstract}
Background and aim: the qualitative and quantitative difference in the intestinal microbiota between irritable bowel syndrome (IBS) patients and healthy volunteers doesn't raise doubts. Small intestinal bacterial overgrowth(SIBO), reported in 9-35\% patients with constipation-predominant of irritable bowel syndrome (IBS-C), is involved in the pathogenesis IBS. Elimination of SIBO can lead to improvement of clinical symptoms. The aim of this study was to assess the efficacy of a bifido- and lactobacillis-containing probiotic Florasan-D on the eradicating SIBO and on the severity of clinical symptoms in patients with IBS-C. Methods: this randomized (1:1), simpleblinded, placebo-controlled investigation conducted at one centre in Russia.33 patients with IBS-C (clinical type was determined according to ROME III criteria) were randomized to receive a multi-strainprobiotic Florasan-D containing Bifidobacteriumbifidum, Bifidobacteriumlongum, Bifidobacteriuminfantis, Lactobacillus rhamnosus or placebo for 4 weeks: 16 and 17 persons respectively. The intensity of abdominal pain and bloating was estimated by a visual analogue scale (VAS), stool consistency - by a Bristol stool form scale before and after the intervention. Quality of life was assessed by the Short Form-36 questionnaire (SF-36). All subjects were analysed by lactulose hydrogen breath test with using Gastro+ Gastrolyzer (Bedfont, UK) to determine SIBO. Results: patients treated with multi-strainprobiotic Florasan-D reported a reduction in the severity of clinical symptoms: abdominal pain decreased from 4 to 2 point $(p=0,003)$, stool frequency - from 3 to 7 per week $(p=0,001)$ and stoolconsistency from type 2 to type 3 by a Bristol stool form scale ( $\mathrm{p}=0,001)$; SIBO was found in $56,3 \%$ patients before treatment and no patients had this syndrome after therapy. Patients treated with placebo reported a reduction the severity of abdominal pain only $(\mathrm{p}=0,028)$. SIBO in these patients before treatment was revealed in $52,9 \%$ and remained in all patients after it. Conclusions: multi-strainprobiotic Florasan-D is significantly more effective than placebo for reduction of clinical symptoms and SIBO elimination in IBS-C patients.
\end{abstract}

Keywords: irritable bowel syndrome, small intestinal bacterial overgrowth, probiotics, treatment

Cite This Article: Ivashkin V., Drapkina O., Poluektova Ye., Kuchumova S., Sheptulin A., and Shifrin O., "The Effect of a Multi-strain Probiotic on the Symptoms and Small Intestinal Bacterial Overgrowth in Constipation-predominant Irritable Bowel Syndrome: A Randomized, Simple-blind, Placebo-controlled Trial.” American Journal of Clinical Medicine Research, vol. 3, no. 2 (2015): 18-23. doi: 10.12691/ajcmr-3-2-1.

\section{Introduction}

Nowadays a role of gastrointestinal microbiota in pathogenesis of irritable bowel syndrome (IBS) is being actively discussed. There is no doubts that there is a qualitative and quantitative difference in the intestinal microbiota between IBS patients and healthy volunteers $[9,15,20,24]$.

Disorder of "bacteria - host" homeostasis is the triggers of the cascade of pathological immune reactions in genetically determined subjects with the changed genes, responsible for barrier function of intestinal epithelium, which leads to clinical manifestation of the disease $[10,18,20,21,24]$.

The revealed changes necessitate a search for more effective schemes for treatment IBS patients, with using medicine for intestinal microbiome normalization.

A number of trials of the assesment of probiotics efficiency in IBS patients are constantly growing. Data on high efficacy of these medicines in IBS patients are contained in the majority of systemic reviews and metaanalyses [2,4,5,6,16].

Unfortunately, in many trials a clinical form of the disease is not specified, bacterial strains, medications dose and duration course of treatment differ greatly, and no 
information about normalization of intestinal microbiota with clinical improvement is available [13].

The above data require further clinical trials to establish the most effective combination of bacterial strains, duration of treatment, and assessment of intestinal microflora before and after therapy.

\section{Materials and Methods}

Randomized (1:1), simple-blinded, placebo-controlled investigation conducted at one centre in Russia. 33 patients with constipation-predominant irritable bowel syndrome (IBS-C) were examined at the Department of Internal Disease Propaedeutics, Gastroenterology and Hepatology of theI.M. Sechenov First Moscow State Medical University.

The diagnosis was confirmed on the base of Rome III criteria and absence of organic diseases according to laboratory and instrumental examinations. The examined group included 5 males (15\%) and 28 females (85\%), average age was 35.0 [33.7; 41.1] years.

We performed a comparative efficiency analysis of a 4week therapy with multi-strainprobiotic Florasan-D (Bifidobacteriumbifidum, Bifidobacteriumlongum, Bifidobacteriuminfantis, Lactobacillusrhamnosus) or placebo with assessment of their influence on severity of the main clinical symptoms, intestinal microflora and quality of life in IBS-C patients.

The patients were divided into 2 subgroups according to their admission to the clinic: 16 patientsreceived multistrainprobiotic Florasan-D, $250 \mathrm{mg}$ twice daily, and 17 patients received placebo (microcrystalline cellulose, lactose, and calcium stearate) in capsules identical to multi-strainprobiotic Florasan-D,250 mg twice daily.
Severity of abdominal pain and bloating was assessed with a visual analogue scale (VAS) in 1 to 10 scores, $10-$ the highest severity of a sign, and $0-$ absence of a sign [27]; disorders of stool consistency - according to the Bristol Stool Form [14]; stool frequency-by patients' count of defecations per week. Symptoms severity was assessed before treatment and at the end of every week of therapy.

A hydrogen breath test with lactulose for exclude the small intestinal bacterial overgrowth (SIBO) syndrome, and quality of life assessment (Short Form-36 (SF-36)) were performed twice (before and after therapy) [26].

Data were analyzed using the Statistica software for Windows version 10.0 (StatSoft Inc.). Qualitative parameters were described by absolute and relative (\%) indices, quantitative parameters - by the median (Me) and 95\% confidence interval (CI): Me [-95\%CI; +95\%CI].To assess statistical confidence of intergroup difference the following parameters were determined: quantitative parameters, 2 independent groups - Mann-Whitney test, quantitative parameters, 2 dependent groups - Wilcoxon test. Kendall's tau correlation was used in correlation analysis. It was assumed that the correlation module was $|\tau| \leq 0.25$ signified weak correlation; $0.25<|\tau|<0.75-$ moderate correlation; $|\tau| \geq 0.75$ - strong correlation. Significance level was considered acceptable atp $<0.05$.

\section{Results}

\subsection{Comparative Analysis of Patients' Subgroups before the Treatment}

Table 1 contains characteristics of IBS-C patient subgroups before the Treatment. Statistically significant difference in bloating severity between the subgroups was revealed.

Table1. Comparative Analysis of Patients' Subgroups before the Therapy

\begin{tabular}{|c|c|c|c|}
\hline Parameters & Patients on multi-strainprobioticFlorasan-D, $n=16$ & Patients on Placebo, n=17 & $\mathbf{P}$ \\
\hline Age (years) & $27.0[24.1 ; 36.8]$ & $34.0[29.4 ; 39.2]$ & 0.201 \\
\hline Age of the debut of a disease & $25.0[19.3 ; 27.7]$ & $25.0[21.2 ; 29.4]$ & 0.815 \\
\hline Duration of Medical History & $6.5[3.6 ; 8.8]$ & $7.0[5.1 ; 13.1]$ & 0.340 \\
\hline Pain (VAS Scale) & $4.0[3.8 ; 5.9]$ & $4.0[3.2 ; 4.7]$ & 0.387 \\
\hline Stool Consistency (Bristol Stool Form) & $2.0[1.8 ; 2.3]$ & $2.0[1.6 ; 2.6]$ & 0.928 \\
\hline StoolFrequency(Defecations/Week) & $3.0[2.4 ; 4.1]$ & $4.0[3.4 ; 4.6]$ & 0.117 \\
\hline Bloating (VAS Scale) & $7.0[6.7 ; 8.7]$ & $6.0[3.6 ; 6.2]$ & $0.003^{*}$ \\
\hline
\end{tabular}

* - Mann-Whitney U Test.

Since the difference in bloating severity between the subgroups was revealed before treatment, we performed a correlation analysis of this parameter with clinical symptoms at the end of the treatment (Table 2) to find out if the severity of bloating had any influence on the treatment results.

Table 2. Correlation between the Main Clinical Symptoms (Week 4 of Therapy) andBaseline Bloating Severity in IBS-C Patients

\begin{tabular}{|l|c|c|}
\hline Parameters & Kendall'stau( $\boldsymbol{\tau})$ & P \\
\hline Abdominal Pain (Week 4) & 0.24 & 0.217 \\
\hline Stool Consistency (Week 4) & -0.01 & 0.948 \\
\hline StoolFrequency (Week 4) & -0.18 & 0.344 \\
\hline Bloating (Week 4) & 0.54 & $0.002 *$ \\
\hline * - Wilcoxon test.
\end{tabular}

We revealed a significant correlation between bloating severity at the baseline and at the end of the treatment. Because of the significant influence of the baseline parameter to the treatment results, we did not compare the treatment efficacy using this parameter.

\subsection{Dynamics of Main Clinical Symptoms in IBS-C Patients using multi-strainprobiotic Florasan-D or Placebo}

Table 3 shows a comparison of the changes of the clinical symptoms which took place each week in Florasan-D and placebo subgroups. Multi-strain probiotic Florasan-D was significantly more efficient in normalasing stool frequency. In view of the baseline difference (Week 0) in bloating severity between the subgroups, no comparison was made between them. 
Table 3. Changes of Clinical Symptoms Severity (Scores) in IBS-C Patients during the treatment with multi-strainprobiotic Florasan-D and Placebo

\begin{tabular}{|c|c|c|c|c|}
\hline \multirow{6}{*}{ 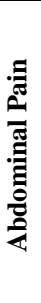 } & $\begin{array}{l}\text { Week of } \\
\text { Therapy } \\
\end{array}$ & $\begin{array}{c}\text { multi-strain } \\
\text { probioticFlorasan-D }\end{array}$ & Placebo & $\mathbf{P}$ \\
\hline & 0 & $4.0[3.8 ; 5.9]$ & $4.0[3.2 ; 4.7]$ & 0.387 \\
\hline & 1 & $4.0[2.6 ; 4.6]$ & $4.0[3.0 ; 4.7]$ & 0.706 \\
\hline & 2 & $3.0[2.2 ; 4.2]$ & $4.0[2.9 ; 4.4]$ & 0.462 \\
\hline & 3 & $2.0[1.4 ; 3.3]$ & $3.0[2.3 ; 4.0]$ & 0.199 \\
\hline & 4 & $2.0[1.2 ; 3.0]$ & $3.0[2.3 ; 3.9]$ & 0.093 \\
\hline \multirow{5}{*}{ 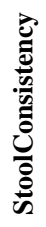 } & 0 & $2.0[1.8 ; 2.3]$ & $2.0[1.6 ; 2.6]$ & 0.928 \\
\hline & 1 & $3.0[2.5 ; 3.5]$ & $2.0[2.0 ; 3.0]$ & 0.177 \\
\hline & 2 & $3.0[2.8 ; 3.7]$ & $3.0[2.3 ; 3.2]$ & 0.180 \\
\hline & 3 & $3.5[3.1 ; 3.9]$ & $3.0[2.4 ; 3.3]$ & 0.082 \\
\hline & 4 & $3.0[2.8 ; 3.7]$ & $3.0[2.1 ; 3.2]$ & 0.126 \\
\hline \multirow{5}{*}{ 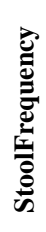 } & 0 & $3.0[2.4 ; 4.1]$ & $4.0[3.4 ; 4.6]$ & 0.117 \\
\hline & 1 & $6.5[5.1 ; 8.5]$ & $4.0[2.3 ; 7.1]$ & 0.008 \\
\hline & 2 & $6.0[5.1 ; 7.3]$ & 5.0 [3.2; 6.3] & $0.033 *$ \\
\hline & 3 & $7.0[4.9 ; 6.7]$ & $4.0[3.0 ; 5.4]$ & 0.050 \\
\hline & 4 & $7.0[5.5 ; 7.5]$ & $4.0[3.8 ; 5.8]$ & $0.016 *$ \\
\hline
\end{tabular}

Table 4 shows difference in symptoms severity during the therapy (Weeks 0 and 4) in every subgroup and comparison between the subgroups.

Both multi-strainprobiotic Florasan-D and placebo were efficient for decreasing of abdominal pain (VAS) at the end of therapy $(p=0.003$ and $p=0.028)$. The decrease of abdominal pain was significantly more pronounced in Florasan-D subgroup $(\mathrm{p}=0.023)$.

Significant improvement of stool consistency according to the Bristol Stool Form Scale was observed at the end of therapy $(\mathrm{p}=0.001)$. No statistically significant difference between the two subgroups was revealed $(\mathrm{p}=0.059)$.

In the Florasan-D subgroup the result that the patients passed stool everyday was achieved $(p=0.001)$. No stool frequency normalization was observed in the placebo subgroup. The subgroup difference was significant $(\mathrm{p}=0.002)$.

By Week 4 the bloating severity (by VAS) was significantly lower compared to that before the therapy in both subgroups $(\mathrm{p}=0.001$ and $\mathrm{p}=0.016)$. Nevertheless, in view of the influence of the baseline bloating to the final results of treatment, no comparison of Florasan-D and placebo subgroups was performed.

Table 4. Difference in Clinical Symptoms Severity during the Therapy (Weeks 0 and 4) in Every Subgroup and Comparison between the Subgroups

\begin{tabular}{|l|c|c|c|}
\hline \multirow{2}{*}{ Parameters } & \multicolumn{2}{|c|}{ P } \\
\cline { 2 - 4 } & multi-strain probioticFlorasan-D & Placebo & multi-strain probioticFlorasan-D/ Placebo \\
\hline Pain (VAS Scale) & $0.003^{*}$ & $0.028^{*}$ & $0.023^{* *}$ \\
\hline Stool Consistency (Bristol Stool Form) & $0.001^{*}$ & $0.046^{*}$ & 0.059 \\
\hline StoolFrequency (Defecations/Week) & $0.001^{*}$ & 0.131 & \\
\hline
\end{tabular}

* - Wilcoxon test ** - Mann-Whitney U Test.
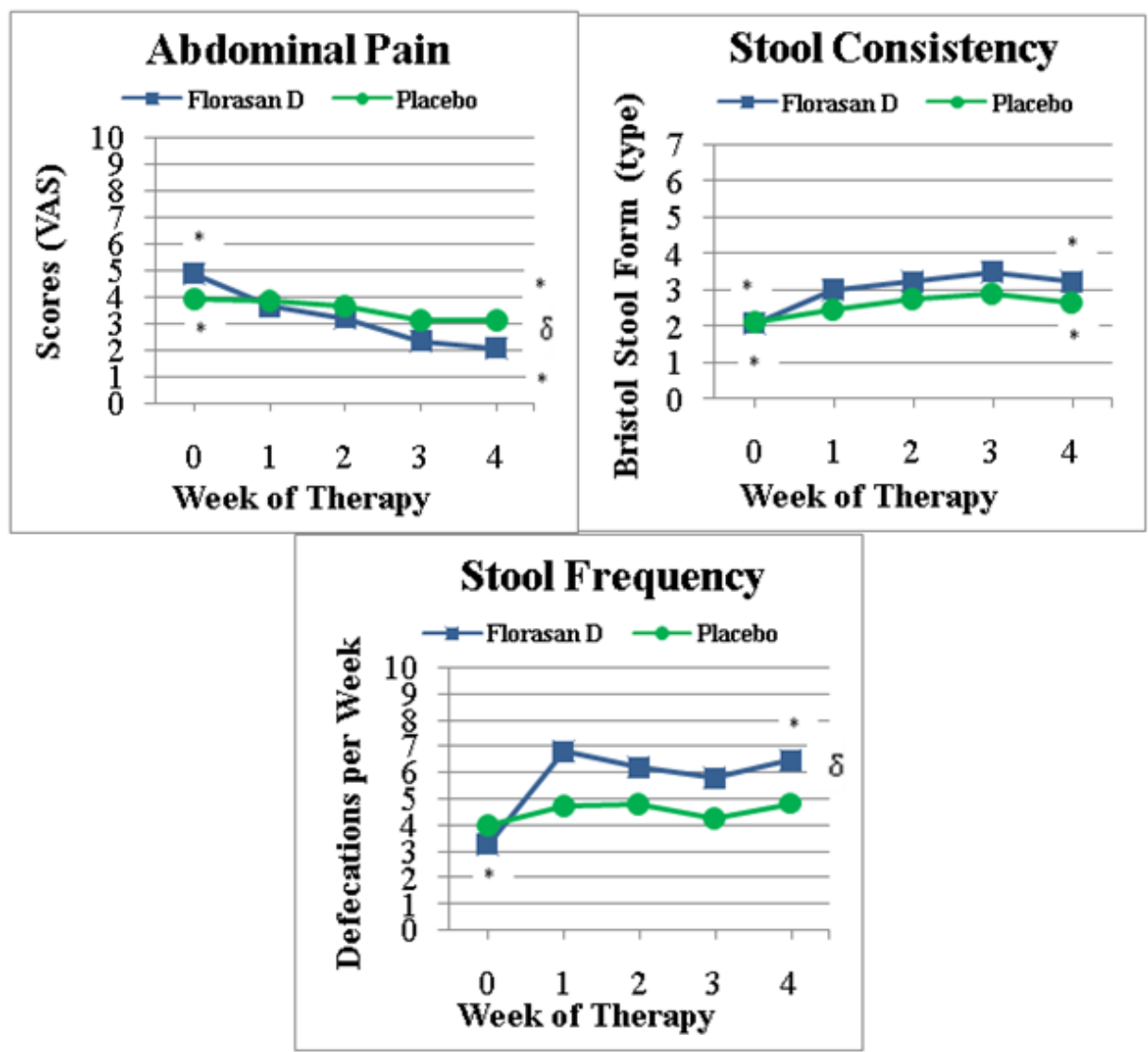

Figure 1. Changes of Clinical Symptoms Severity in patients on multi-strain probioticFlorasan-D or Placebo

*- significant difference in symptom severity (weeks 0 and 4 ) within every subgroup (Wilcoxon test)

$\delta$ - significant difference in symptom severity (weeks 0 and 4) between the subgroups (Mann-Whitney U Test). 
Changes of clinical symptoms severity are shown at Figure 1.

\subsection{Changes of Small Intestinal Bacterial Overgrowth (SIBO) Syndrome in IBS-C Patients Using Multi-strainprobioticFlorasan-D or Placebo}

9 out of 16 patients had SIBO before therapy in the Florasan-D subgroup (56.3\%) (Figure 2). No patients showed SIBO signs after the end of therapy $(\mathrm{p}=0.013)$.

In the placebo subgroup all 9 patients out of 17 patients had SIBO at the baseline (52.9\%) and during the whole 4week treatment period. Difference between the subgroups were significant after the end of the therapy $(p=0.002)$.

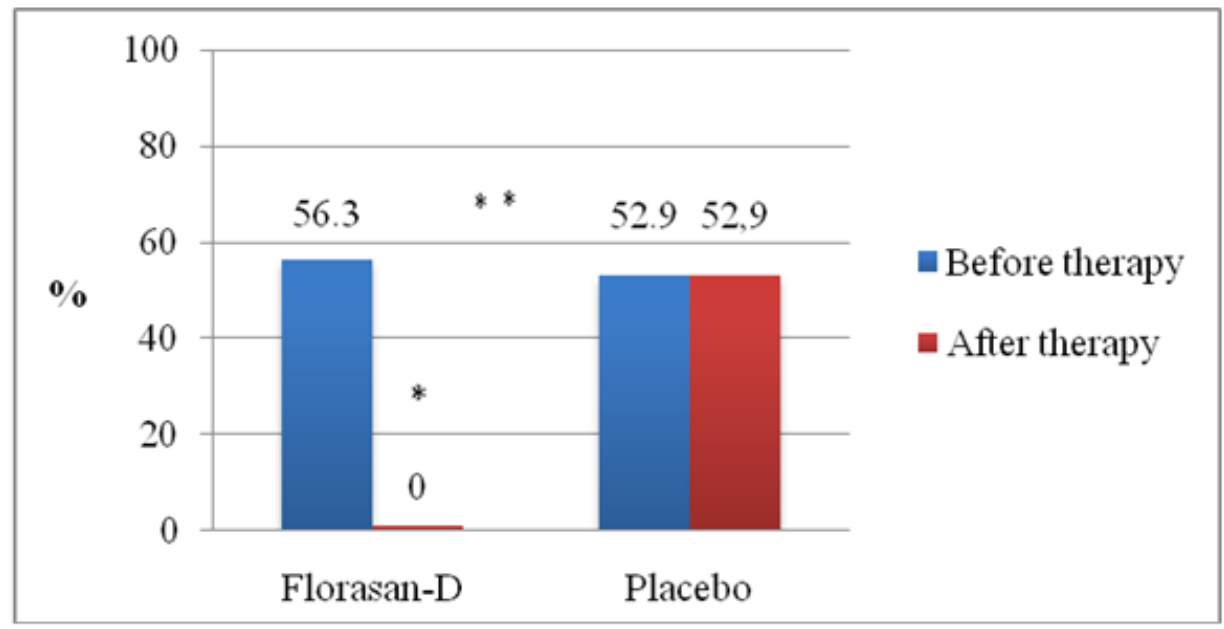

Figure 2. Changes of SIBO Signs in Patients on multi-strain probioticFlorasan-D or Placebo

*- significant difference before and after therapy withFlorasan-D(Wilcoxon test)

** - significant difference between the subgroups (Mann-Whitney U Test).

\subsection{Changes in the Quality of Life (by SF-36 Questionnaire)in IBS-C Patients Using Probiotic Mixture Florasan-D or Placebo}

In the Florasan-D subgroup a significant improvement of the general health $(p=0.027)$ and viability $(p=0.043)$ was observed.

In the placebo subgroup no prominent changes of life quality of life was revealed (Figure 3 ). No significant difference in any parameters before and after therapy was achieved.

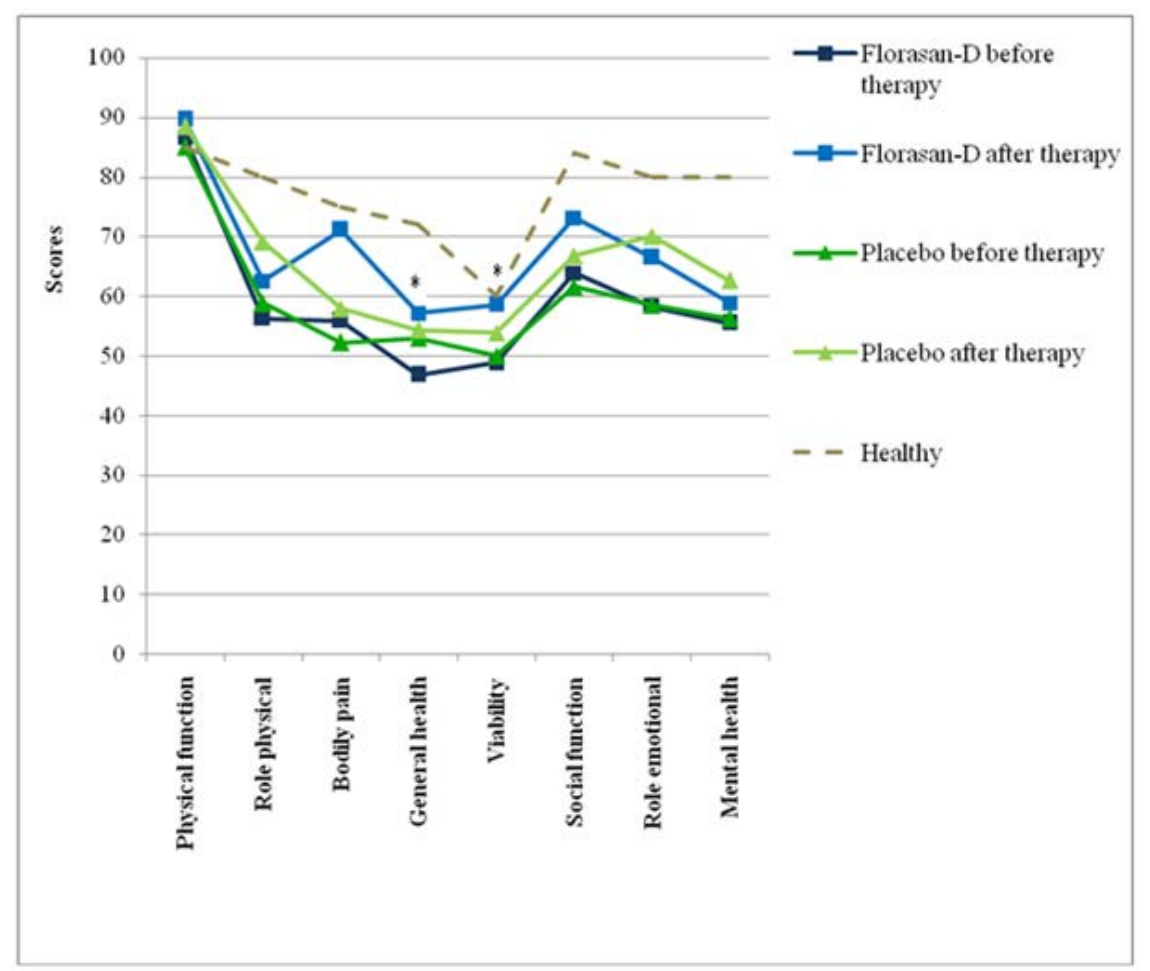

Figure 3. Changes Quality of Life in Healthy Volunteers and IBS-3 Patients before and after multi-strain probioticFlorasan-D or Placebo Therapy *- significant difference before and after therapy with multi-strain probioticFlorasan-D(Wilcoxon test). 
Multi-strain probiotic Probioticmixture Florasan-D showed significantly higher efficacy in the reduction of the main clinical symptoms severity (abdominal pain, stool frequency) and improvement to the quality of the patients' life compared to placebo. Clinical efficiency was associated with the normalization of intestinal microbiota in Florasan-D subgroup.

\section{Discussion}

Genetically determined lower resistance and unbalanced receptiveness to bacterial cell components, disorders of small intestinal peristaltics, and a dysfunctional the ileocecal valve could be grounds for changes of intestinal microbiome in IBS patients [8,22,25,28].

Despite the absence of established opinion on the changes of intestinal microbiota composition typical of diarrhea or constipation-predominantIBS, currently more and more data is been published on the reduction of normal microbiota (Bifidobacteria and Lactobacteria) in such patients [9,15,23]. It is known that Bifidobacteria and Lactobacteriaproduce short-chain fatty acids (SCFA), i.e. oleic acid, acetic acid, and propionic acid, during hydrolytic degradation of carbohydrates. SCFA perform multiple functions such as regulation for intestinal peristaltics and water-electrolytic balance, stimulation for cell differentiation and epithelial regeneration, maintenance for antimicrobial effect due to creation of acidic medium in the bowel lumen [1].

Use of the gas-liquid chromatography proved the reduction in SCFA absolute concentration in IBS-C patients [1]. Quantitative reduction in SCFA could lead to a weakening of epithelial protection and intestinal motility [1], which creates favorable conditions for pathogenic and opportunistic microbiota with adhesion factors.

Penetration of pathogenic and opportunistic microbiota through the epithelial layer triggers a pathway of pathological immune response, intestine wall inflammation, and its peripheral sensitization (i.e. increased perceptivity of nociceptors to subthresholdstimuli), which in turn causes central sensitization and, indirectly, disorder of the intestinal motility $[7,12]$.

Therefore, changes of intestinal microbiome composition (especially decrease in saccharolyticmicroflora) contributes to the maintenance of intestinal wall inflammation and changes of motility and visceral sensitivity causing symptoms in IBS patients.

At present we cannot pharmacologically influence the expression of tight junctions and signaling receptors proteins, nevertheless, in view of multiple influence of inadequate microbiome for clinical symptoms, we consider it reasonable to improve the intestinal microbiota composition by probiotics.

Probiotics facilitate the preservation of intestinal homeostasis [10], synthesize antibacterial substances (bacteriocins, defensins), provide for barrier functions of intestinal epithelium and mucous membranes of the gastrointestinal tract, prevent pathogenic microbiota colonization, enhance local and systemic immune response $[10,11,17,19]$ without any serious side effects [10].

Efficacy of Florasan-D, a probiotic containing the most common representatives of normal intestinal microflora
(Bifidobacteriumbifidum,

Bifidobacteriumlongum, Bifidobacteriuminfantis, Lactobacillusrhamnosus) was studied. These microbial strains have been included in the latest reportby Yale University experts on probiotics use in IBS therapy [3].

On the probiotic mixture Florasan-D therapy, the severity of the main clinical symptoms was reduced significantly, quality of life improved. Suppression of the small intestinal bacterial overgrowth (SIBO) syndrome was revealed by the hydrogen breath test in all patients.

Therefore, probiotics containing a complex of Bifidobacteria and Lactobacteria can be used as monotherapy in IBS-C patients.

We propose that probiotics allow breaking circulusvitiosus which includes peripheral and central sensitization, motility disorders, and symptom expression. Nevertheless, additional studies are necessary to confirm this hypothesis.

\section{Conclusions}

1. Correlation of IBS pathogenesis and clinical symptoms with changes of intestinal microbiota composition makes the use of probiotics in such patients reasonable.

2. Using ofmulti-strain probioticFlorasan-D (Bifidobacteriumbifidum, Bifidobacteriumlongum, Bifidobacteriuminfantis, Lactobacillus rhamnosus) in IBS-C patients for 4 weeks leads to significant reduction in abdominal pain, increase of stool frequency and quality of life, normalization of intestinal microbiome composition compared to placebo.

3. Florasan-D (Bifidobacteriumbifidum, Bifidobacteriumlongum, Bifidobacteriuminfantis, Lactobacillus rhamnosus) was well tolerated by the patients and did not cause any serious side effects.

\section{Funding and Conflict of Interest}

The authors declared that they do not have anything to disclose regarding funding or conflict of interest with respect to this manuscript.

\section{References}

[1] Ardatskaya M.F. The clinical significance of short-chain fatty acids in the pathology of gastrointestinal tract, thesis for a Doctor's degree, Moscow, 2006.

[2] Clarke G., Cryan J.F., Dinan T.G., Quigley E.M. Review article: probiotics for the treatment of irritable bowel syndrome-focus on lacticacid bacteria. Aliment PharmacolTher, 35(4): 403-13; Feb 2012.

[3] Floch M.H., Walker W.A., Madsen K., Sanders M.E., Macfarlane G.T., Flint H.J., Dieleman L.A., Ringel Y., Guandalini S., Kelly C.P., Brandt L.J. Recommendations for probiotic use - 2011 update. J ClinGastroenterol, 45: S. 168-71; 2011.

[4] Ford A.C., Quigley E.M., Lacy B.E., Lembo A.J. et al. Efficacy of Prebiotics, Probiotics, and Synbiotics in Irritable Bowel Syndrome and Chronic Idiopathic Constipation: Systematic Review and Meta-analysis.Am J Gastroenterol,109(10): 1547-61; 2014.

[5] Hosseini A., Nikfar S., Abdollahi M. Probiotics use to treat irritable bowel syndrome. Expert OpinBiolTher, 12: 1323-34; 2012. 
[6] Hoveyda N., Heneghan C., Mahtani K.R., Perera R., Roberts N., Glasziou P. A systematic review and meta-analysis: probiotics in the treatment of irritable bowel syndrome. BMC Gastroenterol, 9: 15; 2009.

[7] Ivashkin V.T., PolouektovaYe. A. Functional gastrontestinal disorders, Moscow, 2013.

[8] Kellow J.E., Eckersley G.M., Jones M. Enteric and central contributions to intestinal dysmotility in irritable bowel syndrome. Dig Dis Sci, 37: 168-74; 1992.

[9] Kerckhoffs A.P., Samsom M., Van der Rest M.E. et al. De Vogel J., Knol J., Ben-Amor K., Akkermans L.M. Lower Bifidobacteria counts in both duodenal mucosa-associated and fecal microbiota in irritable bowel syndrome patients. World J Gastroenterol, 15: 2887-92; 2009.

[10] Khan M.W., Kale A.A., Bere P., Vajjala S., Gounaris E., Pakanati K.C. Microbes, intestinal inflammation and probiotics. Expert Rev GastroenterolHepatol, 6: 81-94; 2012.

[11] KuchumovaS.Yu., PoluektovaYe.A., Sheptulin A.A., Ivashkin V.T. Physiogical value of intestinal microflora, RJGHC, 21(5): 17-27; 2011.

[12] KurbatovaA.A. Pathogenetic and clinical significance of system cytokines and claudins in patients with irritable bowel syndrome, thesis for a master's degree, Moscow, 2013.

[13] Lee K.N., Lee O.Y. Intestinal microbiota in pathophysiology and management of irritable bowel syndrome. World J Gastroenterol. 21: 20-27; 2014.

[14] Lewis S.J., Heaton K.W. Stool form scale as a useful guide to intestinal transit time. Scand J Gastroenterol, 32(9): 920-24; 1997.

[15] Malinen E., Rinttilä T., Kajander K., Mättö J., Kassinen A., Krogius L., Saarela M., Korpela R., Palva A.Analysis of the microbiota of irritable bowel syndrome patients and healthy controls with real-time PCR. Am J Gastroenterol, 100: 373-82; 2005.

[16] Moayyedi P., Ford A. Talley N.J., Cremonini F., Foxx-Orenstein A.E., Brandt L.J., Quigley E.M. The efficacy of probiotics in the treatment of irritable bowel syndrome: a systematic review. Gut, 59: 325-32; 2010.
[17] Ng S.C., Hart A.L., Kamm M.A., Stagg A.J., Knight S.C. Mechanisms of action of probiotics: recent advances. Inflamm Bowel Dis, 15: 300-10; 2009.

[18] Nucera G., Gabrielli M., Lupascu A., Lauritano E.C., Santoliquido A. et al. Abnormal breath tests to lactose, fructose and sorbitol in irritable bowel syndrome may be explained by small intestinal bacterial overgrowth. Aliment PharmacolTher, 21: 1391-5; 2005.

[19] OhlandC.,MacNaughtonK. Probiotic bacteria and intestinal epithelial barrier function. Am J PhysiolGastrointest Liver Physiol, 298(6): 807-19; 2010.

[20] Pimentel M., Chow E.J., Lin H.C. Normalization of lactulose breath testing correlates with symptom improvement in irritable bowel syndrome. A double-blind, randomized, placebo-controlled study. Am J Gastroenterol,98: 412-9; 2003.

[21] PoluektovaYe. A., KuchumovaS.Yu., Shifrin O.S., Sheptulin A.A., Ivashkin V.T. Pathogenic role of intestinal microflora changes in patients with irritable bowel syndrome and treatment options, RJGHC, 24(3):89-97; 2014.

[22] Quigley E.M. Intestinal dysmotility and the irritable bowel syndrome. Ir J Med Sci, 163: 560-1; 2004.

[23] Rajilić-StojanovićM., Biagi E., Heilig H.G., Kajander K., Kekkonen R.A., Tims S., de Vos W.M. Global and deep molecular analysis of microbiota signatures in fecal samples from patients with irritable bowel syndrome. Gastroenterology, 141: 1792-1801; 2011.

[24] Shah E.D., Basseri R.J., Chong K., Pimentel M. Abnormal breath testing in IBS: a meta-analysis. Dig Dis Sci, 55: 2441-9; 2010.

[25] Vorobyev A.A. Microbiology and immunology, Moscow, 1999.

[26] Ware J.E., Snow K.K., Kosinski M., Gandek B. SF-36 Health Survey. Manual and interpretation guide. The Health Institute, New England Medical Center. Boston, Mass, 1993.

[27] Weaver M.E., Lowe N.K. A critical review of visual analogue scales in the measurement of clinical phenomena. Res Nurs Health, 13(4): P. 227-36; 1990.

[28] Zvyagintseva T.D., Gridneva S.V. Irritable bowel syndrome, disbiosis, Enterogermina. News of medicine and pharmacy, 17(291); 2009. 